\title{
A Risk-Based Diagnostic Campaign Optimization For Electric Power Distribution Networks
}

\author{
Luca Bellani, Roberto Mascherona \\ Aramis s.r.l., Milano, Italy.E-mail: luca.bellani@aramis3d.com,roberto.mascherona@aramis3d.com
}

\author{
Michele Compare \\ Aramis s.r.l., Milano, Italy. E-mail: michele.compare@aramis3d.com \\ Energy Department, Politecnico di Milano, Italy

\section{Enrico Zio} \\ Aramis s.r.l., Milano, Italy \\ Energy Department, Politecnico di Milano, Italy.E-mail: enrico.zio@polimi.it \\ MINES ParisTech, PSL Research University, CRC, Sophia Antipolis, France \\ Eminent Scholar, Department of Nuclear Engineering, College of Engineering, Kyung Hee University, \\ Republic of Korea \\ Gaetano Iannarelli \\ Department of Astronautics, Electrical and Energy Engineering, Università degli Studi La Sapienza, Roma, \\ Italy \\ UNARETI S.p.A., Milano, Italy E-mail: gaetano.iannarelli@unareti.it
}

Vittorio Giarola, Andrea Ruffini, Bartolomeo Greco, Andrea Morotti, Luca Cavalletto

UNARETI S.p.A., Milano, Italy E-mail:vittorio.giarola@unareti.it, andrea.ruffini@unareti.it, bartolomeo.greco@unareti.it, andrea.morotti@unareti.it,luca.cavalletto@unareti.it

\author{
Manuel Intini \\ a2a S.p.A., Milano, Italy, E-mail: manuel.intini@a2a.eu
}

Failures of Medium Voltage Distribution Networks (MVDN) can result in power outages, penalties from authorities and large operational costs. To prevent the network failures, diagnostic campaigns are performed to estimate the degradation state of its branches. For underground MVDN, diagnostic mainly relies on Partial Discharge (PD) and Tan-delta measurements. These are costly and, thus, only a small portion of the network can be analyzed every year. Therefore, the prioritization of the PD measurements on the MVDN branches is fundamental for its efficient management. Within the Portfolio Decision Analysis, we develop a risk-based methodology for the identification of the optimal portfolios of MVDN branches undergoing diagnostic measurements. The branch failure likelihood is estimated through an expert-driven model based on the Multi Attribute Value Theory, while accommodating the incomplete information about the impact that network features have on the degradation mechanisms affecting the cable joints and insulators. The severity accounts for the number of users disconnected upon failure, which depends on the topology of the network. The optimal portfolio of inspections is obtained from an algorithm of the literature. Results of the method on a real industrial case study concerning the network of the Milan area are discussed.

Keywords: Power Distribution System, Partial Discharges, Portfolio Decision Analysis, Risk-Based Maintenance

\section{Introduction}

A correct diagnosis of the health state of the electrical cables of underground Medium Voltage Distribution Networks (MVDNs) allows estimating their times to failures and, on this basis, optimizing maintenance. Nowadays, Partial Discharge
(PD) measurements are widely used for diagnostics of network branches (i.e., the MVDN elements connecting two distribution substations), as they provide reliable information about the presence of localized defects. PD measurements are often corroborated by Tan-delta measurements, which estimate the dielectric condition of the

Proceedings of the 30th European Safety and Reliability Conference and the 15th Probabilistic Safety Assessment and Management Conference Edited by Piero Baraldi, Francesco Di Maio and Enrico Zio Copyright (C) ESREL2020-PSAM15 Organizers.Published by Research Publishing, Singapore. 
overall branch.

Performing PD and Tan-delta measurements, however, requires specialized crews with costly measurement facilities and, also, the unavailability of the measured branch for a few hours. From this, it emerges that cable diagnostics is very expensive and can be performed on a very limited portion of the MVDN every year. In practice, maintenance budgets are such that a prioritization of the diagnostic activity on the MVDN is required.

We formalize this maintenance budget allocation issue on critical infrastructures as a Multi-Criteria Decision Making (MCDM) problem (e.g., Keeney and Raiffa (1993)). For instance, MCDM models have been applied in Hilber et al. (2007) to balance preventive and corrective maintenance on a MVDN in order to obtain the lowest total cost, System Average Interruption Frequency Index (SAIFI) and the System Average Interruption Duration Index (SAIDI) Billinton (1996).

Despite the successful application to literature case studies, MCDM models find limited use in real MVDN contexts, because they are unable to address some relevant practical problems such as:

- handle the incomplete knowledge of the parameters influencing network operations and, thus, the values of the diagnostic tasks (here referred to as projects) with respect to the criteria;

- accommodate the imprecision in the decision maker's preference statements about the importance of the decision criteria;

- consider synergies among the projects and other mutual inter-dependencies or constraints (e.g., the minimum amount of projects per area);

- use optimization algorithms capable of identifying non-dominated project portfolios from a large (i.e., a few thousands) set of candidate alternatives.

The aforementioned issues can be effectively addressed by the Robust Portfolio Modeling (RPM) technique (Liesiö et al. (2007, 2008)), which has been successfully applied in Mild et al. (2015) to select cost-effective portfolios (i.e., sets) of projects in support to maintenance budget allocation for transportation networks. Moreover, the non-deterministic optimization algorithm developed in Mild et al. (2015) can identify subsets of the portfolio Pareto set, in acceptable computational times. RPM has also been applied in Mancuso et al. (2016) for maintenance budget allocation in sewerage networks, where a RiskBased Maintenance (RBM) approach has been developed (Dey (2001); Dey et al. (2004)): maintenance efforts are allocated on the considered system to minimize its residual risk of failure.
By embedding the RBM approach within RPM, resources can be cost-effectively allocated to provide a high priority to high-risk items, while still guaranteeing the adequate effort on low-risk items.

Building on Mancuso et al. (2016), RPM has been applied in Sacco et al. (2019) for RBM of pipelines for a realistic model of the Great Britain high-pressure National Gas Network (NGN).

In this paper, we propose a RBM framework to support Decision Makers (DMs) in the identification of optimal PD and Tan-delta diagnostic campaign on MVDNs. The framework applies RPM to identify those diagnostic measurements (i.e., actions) which can reduce most the severity and likelihood of failures in the MVDN.

The paper is organized as follows. In Section 2, we briefly introduce the risk-based maintenance framework. In Section 3, we outline the procedure to quantify the contributions of MVDN branch features to the failure likelihood. In Section 4, we report the optimization problem and the solution algorithm. In Section 5, the presented framework is applied to the MVDN of the city of Milan, Italy. The failure likelihood model is validated through a comparison with the failures recorded in a proper time window. In Section 6, conclusions are drawn.

\section{Risk-based maintenance of large MVDN}

Building on Mancuso et al. (2016), the allocation of PD and Tan-delta diagnostics on MVDN is framed as a two-objective decision problem, the objectives being the two risk factors: failure likelihood and failure severity.

To identify the MVDN branches with the largest failure likelihood, we apply the Multi Attribute Value Theory (MAVT, Keeney and Raiffa (1993)). Generally, this is based on the identification of the relevant attributes of the objective, which are organized in a hierarchical structure: the overall objective (i.e., failure likelihood) depends on relevant high level attributes, which are further decomposed into more detailed sub-attributes. The decomposition is iterated until the lowest level is reached. This contains the leaf attributes, with regard to which the alternative projects (i.e., diagnostic tasks) can be meaningfully evaluated.

Due to incomplete knowledge on the MVDN technical and operational parameters, the scores of the alternatives with respect to the leaf attributes are affected by uncertainties, represented as intervals of possible values.

RPM is applied to identify the portfolios of MVDN branches which have the largest impact on risk reduction. Details of the RPM approach are provided in Mild et al. (2015). 


\section{Electrical line failure probability and severity}

Based on a series of interviews, a team of experts reported that the number and the characteristics of the joints connecting the different cables are the attributes most relevant for the branch failure likelihood.

According to the experts, the joint failure likelihood depends on the material of the insulator of the weakest cable out of the two it connects. Then, a ranking of these material has been provided by the experts, so that material of type $c \in$ $\{1, \ldots, C-1\}$ entails a larger failure likelihood than material of type $c+1$ :

- $c=1$ : Paper not impregnated;

- $c=2$ : Paper impregnated with a migrating mixture;

- $c=3$ : Paper impregnated with not migrating mixture;

- $c=4$ : Paper impregnated with stabilized mixture;

- $c=5$ : Insulating compound based on EPR rubber;

- $c=6$ : Insulation compound based on high modulus EPR rubber;

- $c=7$ : Insulating compound based on cross-linked polythene;

- ...

For example, if a joint connects a cable of type $c_{1}$ and a cable of type $c_{2}, c_{2}>c_{1}$, the resulting failure likelihood of the joint depends only on $c_{1}$. To simplify the notation, in this case we state that the joint is of level $c_{1}$.

We applied the SMART/SWING rating technique Mustajoki et al. (2005) to elicit from experts the upper bounds of the relative importance of the contributions to the failure likelihood of the different types of joints. This yielded scores $\left\{q_{1}, q_{2}, \ldots, q_{C}\right\}$, such that $q_{1}=100, q_{C}=0$, $q_{i}>q_{i+1} \forall i \in\{1, \ldots, C\}$. From the interviews with experts, it also emerged that:

- Statement $s_{1}$ : the presence of at least a joint of type $c_{1}$ is more risky than the presence of joints of type $c_{2}, c_{2}>c_{1}$, independently on the number of joints of type $c_{2}$;

- Statement $s_{2}$ : the risk of the branch increases with the number of joints.

Based on these considerations, each network branch $b$ in $\mathcal{B}=\{1, \ldots, B\}$ has two leaf attributes for each type of joint, i.e.:

- $v_{c}^{b}$, which is the number of joints of level $c$ in network branch $b$. This value is normalized to the maximum number of joints in a branch throughout the network;
- $x_{c}^{b}$, which is the binary indicator of the presence of at least a joint of type $c$ in network branch $b$.

This entails that the there are $2 \cdot C$ leaf attributes (Figure 1).

The failure likelihood $V_{L}(b)$ of branch $b$ is estimated through the following weighted sum:

$$
V_{L}(b)=\sum_{c=1}^{C}\left(w_{c, 1} \cdot v_{c}^{b}+w_{c, 2} \cdot x_{c}^{b}\right)
$$

The weights in Equation 1 represent the preferences elicited from experts. They must sum to one (Equations 2-3) and fulfill statements $s_{1}$ and $s_{2}$ (Inequalities 4 and 5 , respectively). Finally, Inequalities 6 represent the outcomes of the SMART/SWING approach.

For brevity, the weights in Equation 1 are arranged in vector $\mathbf{w}=\left[w_{1,1}, w_{1,2}, \ldots, w_{C, 1}, w_{C, 2}\right]$. Inequalities 4-6 do not allow obtaining a single value for the weights; rather, they identify a region $\mathcal{W} \subset[0,1]^{2 \cdot C}$ which the weights must belong to Salo and Hämäläinen (1992); Mustajoki et al. (2005). The uncertainty in the attribute weights must be propagated through Equation 1, to get the uncertain value of $V_{L}(b) \in\left[\underline{V}_{L}(b), \bar{V}_{L}(b)\right]$, where:

$$
\begin{aligned}
& \underline{V}_{L}(b)=\min _{\mathbf{w} \in \mathcal{W}} \sum_{c=1}^{C}\left(w_{c, 1} \cdot v_{c}^{b}+w_{c, 2} \cdot x_{c}^{b}\right) \\
& \bar{V}_{L}(b)=\max _{\mathbf{w} \in \mathcal{W}} \sum_{c=1}^{C}\left(w_{c, 1} \cdot v_{c}^{b}+w_{c, 2} \cdot x_{c}^{b}\right)
\end{aligned}
$$

The severity of the failure $V_{S}(b)$ depends on the number of users disconnected upon failure of the branch: even if some portions of the network can be supplied by other substations, when a branch fails all the users served by the feeder containing it are disconnected. Notice that to reduce the computational burden, we do not take into account other network features (e.g., topology of switchers and connections, the time to restore the supply in the network) requires). These will be considered in future research works.

\section{Optimization}

The diagnostic allocation problem is framed as an optimization problem within the Portfolio Decision Analysis (PDA Salo et al. (2011)): we have to identify the optimal portfolios of diagnostic tasks which allow maximizing the total failure likelihood and the total failure severity. The optimization problem is constrained: the inspection of a branch $b$ entails a cost $c(b)$ and the available 


$$
\begin{array}{cl}
w_{c, 1}, w_{c, 2} \in[0,1] & \\
\sum_{c=1}^{C}\left(w_{c, 1}+w_{c, 2}\right)=1 & \\
w_{c_{1}, 2} \geq \sum_{c=c_{1}+1}^{C}\left(w_{c, 1}+w_{c, 2}\right), & c_{1} \in\{1, \ldots, C-1\} \\
w_{c, 1} \geq w_{c, 2}, & c \in\{1, \ldots, C-1\} \\
w_{c_{1}, 2} \cdot q_{c_{2}} \geq w_{c_{2}, 2} \cdot q_{c_{1}} & c_{1}, c_{2} \in\{1, \ldots, C\}, c_{2}>c_{1}
\end{array}
$$

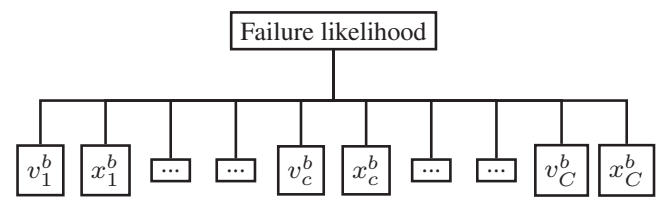

Fig. 1.: Failure likelihood tree

budget is $\beta$. We resort to RPM (Liesiö et al. (2007, 2008); Mild et al. (2015)) to solve this optimization problem.

Notice also that it is possible to incorporate synergies and/or logic synergies constraints for the inspection activities, e.g., the diagnostic action on two neighbor branches belonging to the same feeder may cost a percentage $p \in(0,1)$ less than the sum of the individual inspection costs (Liesio et al. (2007, 2008); Mild et al. (2015)).

The overall value $V(F)$ of a portfolio $F \in \mathcal{F}$ is the sum of the values of the failure likelihood and severity of all the branches belonging to $F$. If $z_{b}(F)$ is the binary indicator variable such that $z_{b}(F)=1$ iff $b \in F$, then:

$$
V(F)=\boldsymbol{\omega}^{T} \cdot S \cdot \mathbf{z}(F)
$$

where

- $\boldsymbol{\omega}^{T}=\left[\omega_{1}, \omega_{2}\right] \in Z_{\Omega} \subset \mathbb{R}^{2}, \omega_{1}, \omega_{2} \in$ $[0,1], \omega_{1}+\omega_{2}=1$ are the weights that describe the relative importance of the two objectives

- $S \in Z_{S} \subset \mathbb{R}^{2 \times B}$ is the score matrix, such that $S_{1, b}=V_{L}(b)$ and $S_{2, b}=V_{S}(b)$ $\forall b \in \mathcal{B}$ respectively

- $\mathbf{z}(F) \in \mathbb{R}^{B}$ contains $z_{b}(F), \forall b \in \mathcal{B}$

Under incomplete information about attribute weights $\omega$ and scores $S, V(F)$ is also uncertain. We state that portfolio $F_{1}$ dominates $F_{2}$ if $F_{1}$ has an overall value larger than or equal to that of $F_{2}$ for all feasible attributes weights and scores and strictly larger for at least one combination of attribute weights $\omega$ and scores $S$, i.e.:

$$
\begin{cases}V\left(F_{1}\right) \geq V\left(F_{2}\right) & \text { for all } S, \omega \in Z_{S} \times Z_{\Omega} \\ V\left(F_{1}\right)>V\left(F_{2}\right) & \text { for some } S, \omega \in Z_{S} \times Z_{\Omega}\end{cases}
$$

Then, the set of optimal portfolios $\mathcal{F}^{*} \subset \mathcal{F}$ is obtained by solving the optimization problem

$$
\arg \max _{F \in \mathcal{F}} \boldsymbol{\omega}^{T} \cdot S \cdot \mathbf{z}(F) \text { s.t. } \mathbf{c}^{T} \cdot \mathbf{z}(F) \leq \beta
$$

where $\mathbf{c} \in \mathbb{R}^{B}$ contains the cost values $\left(c_{b}=\right.$ $c(b))$.

The optimization problem we are tackling is very complex, as the search space of the possible inspection portfolios contains $2^{B}$ solutions, with $B>1$ (e.g., $B>1000$ ). Consequently, the exact dynamic programming algorithms proposed in Liesiö et al. $(2007,2008)$ are not applicable. Therefore, we use its extension developed in Mild et al. (2015), which finds portfolios that are cerytainly optimal, although the algorithm may not find all optimal portfolios.

To analyze the solutions provided by the algorithm, we consider the Core Index (CI) metric. The CI of branch $b$ is defined as:

$$
C I(b)=\frac{\mid\left\{F \in \mathcal{F}^{*} \text { s.t. } b \in F\right\} \mid}{\left|\mathcal{F}^{*}\right|}
$$

where $|\square|$ denotes the number of elements in set $\square$. In words, $C I(b)$ represents the portion of optimal portfolios including diagnostic on branch $b$. When the CI of a branch is 1 , then the branch is included in all the identified optimal portfolios. We indicate by $\mathcal{B}^{+}=\{b \in \mathcal{B}$ s.t. $C I(b)=1\}$ the set containig all the branches with $C I$ equal to 1 . Conversely, when the CI of a branch is 0 , then it is included in none of the identified optimal portfolios: $\mathcal{B}^{-}=\{b \in \mathcal{B}$ s.t. $C I(b)=0\}$ contains all the branches with $\mathrm{CI}$ equal to 0 .

\section{Case study}

In this Section, we apply the developed procedure to the MVDN of the city of Milan, Italy. This is made up of $B=5361$ branches belonging to 606 different feeders. 
According to the experts, there are $C=7$ different insulator types (and, thus, of joint failure likelihood), with relative risk contribution $q_{1}=$ $100, q_{2}=95, q_{3}=80, q_{4}=60, q_{5}=50, q_{6}=$ $5, q_{7}=0$.

The inspection cost does not depend on the network branches. Thus, the constraint is translated in terms of maximum number of inspections to perform in a chosen period, which depends on the number of people and resources available to perform the diagnostics activity. In formulas, $c(b)=1 \forall b \in\{1, \ldots, 5361\}$, whereas $\beta=1500$. This corresponds to a time horizon of almost five years.

The RPM algorithm was set to stop when the difference of each branch CI among the last 100 iterations was lower than $1 \%$. This took about 5 minutes on a 4-core computer with 32GB RAM.

Figure 2 depicts the lower and upper bounds of the failure likelihood $V_{L}(b)$ and $\bar{V}_{L}(b)$ for all the branches of the Milan MVDN. The branches are sorted according to the number of joints with decreasing failure likelihood: we first sort the branches based on $v_{1}^{b}$ (i.e., the number of joints belonging to class 1 ), then we look at $v_{2}^{b}$ (the number of joints of class 2 (i.e., those associated with relative importance weight $q_{2}=95$ ) and so on. From the analysis of the Figure, we can see that both $V_{L}(b)$ and $\bar{V}_{L}(b)$ monotonically decrease with the index of the branches, which is in accordance to the experts' preference statements. Moreover, there are 1708 branches with at least one joint of class 1 , which entail that $V_{L}(b)>0$ $\forall b \leq 1708$ and 936 branches with no joints, which have $\bar{V}_{L}(b)=0$. Notice that the large uncertainty in the values of $V_{L}(b)$ arises from the large uncertainty in the preference statements of the experts through Inequalities 6.

Figure 3 reports the CI resulting from the opti-

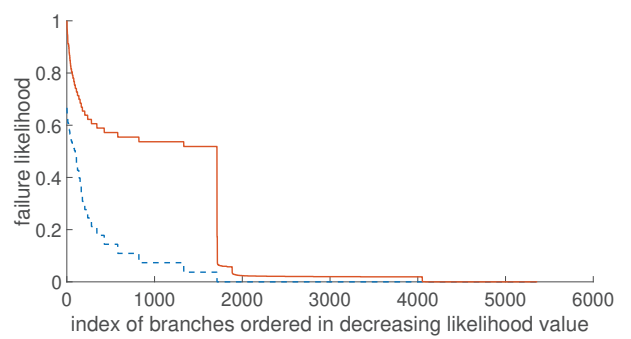

Fig. 2.: $V_{L}(b)$ and $\bar{V}_{L}(b)$ for all the branches of Milan MVDN

mization algorithm for all the branches of the considered MVDN. For visualization, the branches have been sorted in decreasing order of CI value (i.e., the branches are sorted in a different order with respect to Figure 2). From the analysis of the Figure, we can see that there are only 2830 branches with $C I>0$, which means that the remaining 2531 are not included in any project. Although the optimal solution should be selected among the set of the optimal portfolios using different criteria such as the maximin or minimax regret rules (Liesiö et al. (2007)), the core index may be an interesting metric to identify the core branches. For instance, from Figure 3, we can see that 146 portfolios have a $C I=1$ (i.e., $\left|\mathcal{B}^{+}\right|=$ 146): these portfolios could be inspected in the first months of the analysis, thus giving time to reschedule the tasks.

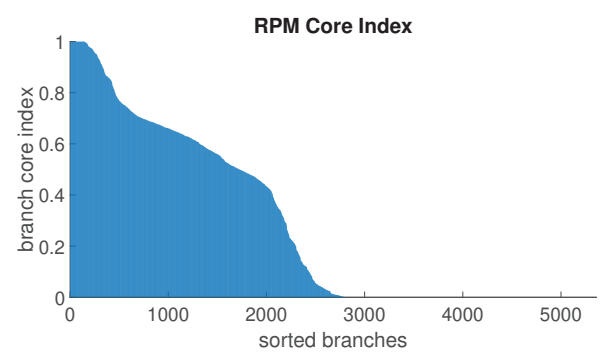

Fig. 3.: Core Index for all the branches of Milan MVDN

\subsection{Model validation}

The validation of the proposed methodology relies on two steps: the evaluation of the failure likelihood model and that of the optimization algorithm.

To assess the quality of the proposed failure likelihood estimation methodology, we can compare the values of $V_{L}(b)$ with the historical data of failures. The drawback of this approach lies in that upon failure of a branch $b$, its joint and cables are changed, leading to different values of $x_{c}^{b}$ and $v_{c}(b) \forall c \in\{1, \ldots, C=7\}$ and, thus, of $V_{L}(b)$ itself. This entails that for a fair comparison, we can consider the failures occurred over a short time horizon, only. This weakens the confidence on the results.

We considered the data of 3 months. For confidentiality, we cannot give information concerning the total number of failures. The results of the validation procedure are summarized in Table 1 . Namely, we sort the branches according to $\bar{V}_{L}(b)$ and we divide the branches in groups of 50 to 100 elements as reported in row 1 (this entails, for example, that column 2 of Table 1 refers to branches 1 to 50 , i.e., the 50 branches with largest $\bar{V}_{L}(b)$ value). The second and the third rows report, respectively, the percentage of failures 
that occurred on the branches of each group and the corresponding cumulative number of failures (e.g., row 3, column 3 entails that branches 1 to 100 have experienced $12.8 \%$ of the total failures). From the analysis of the Table, we can see that:

- more than $7 \%$ of the failures occurred on the 50 branches with largest $\bar{V}_{L}(b)$ (i.e, less than $1 \%$ of the total network);

- almost $13 \%$ of failures occurred on the first 100 branches (i.e, less than $2 \%$ of the total network);

- more than $30 \%$ of failures occurred on the first 500 branches (i.e, less than $10 \%$ of the total network);

- the percentage of failures in the various groups seems to decrease with the value of $\bar{V}_{L}(b)$.

Moreover, the branches with no joints (which roughly are $20 \%$ of the total number of branches) experienced only $0.4 \%$ of the total failures. This suggests that the failure likelihood model is capable of identifying the most critical branches.

With respect to the optimization algorithm, we assessed the stability of the algorithm by performing several runs and verifying that the CI of the different branches used to stabilize more or less always after the same amount of iterations (i.e., about 1000 ). In particular, we verified that the set $\mathcal{B}^{+}$of core branches and the set $\mathcal{B}^{-}$of exterior branches were the same over all runs.

Finally, in Figure 4, we report the total severity and the failure likelihood of all the computed optimal portfolios. The Figure shows that the algorithm has explored uniformly the set of the nondominated solutions (i.e., it has explored different combinations of the weights $\boldsymbol{\omega}$ so as to consider both objectives).

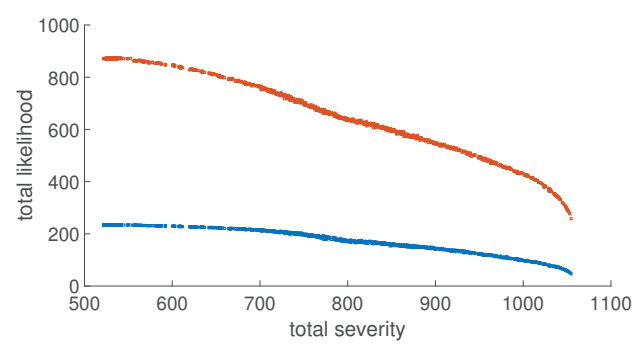

Fig. 4.: Pareto front for all the branches of Milan MVDN

\section{Conclusions}

In this work, we have proposed a risk-based methodology to select the portfolios of MVDN branches to prioritize in the PD diagnostic measurements of underground MVDNs. The failure likelihood is estimated through an expert driven model, which accounts for the number and insulator materials of the joints and cables of the branch, whereas the severity accounts for the number of users disconnected upon failure. An effective optimization algorithm has been taken from literature. The application of the proposed framework to the Milan MVDN has shown that the likelihood values identified with our model are in accordance with the failures experienced by the network branches. The optimization algorithm has been able to converge to a stable set of core indexes in about 1000 iterations.

\section{References}

Billinton, R. (1996). Reliability evaluation of engineering systems. Springer.

Dey, P. (2001). A risk-based model for inspection and maintenance of cross-country petroleum pipeline. Journal of Quality in Maintenance Engineering 7(1), 25-41.

Dey, P., S. Ogunlana, and S. Naksuksakul (2004). Risk-based maintenance model for offshore oil and gas pipelines: A case study. Journal of Quality in Maintenance Engineering 10(3), 169-183.

Hilber, P., V. Miranda, M. A. Matos, and L. Bertling (2007, Nov). Multiobjective optimization applied to maintenance policy for electrical networks. IEEE Transactions on Power Systems 22(4), 1675-1682.

Keeney, R. L. and H. Raiffa (1993). Decisions with multiple objectives: preferences and value trade-offs. Cambridge university press.

Liesiö, J., P. Mild, and A. Salo (2007). Preference programming for robust portfolio modeling and project selection. European Journal of Operational Research 181(3), 1488-1505.

Liesiö, J., P. Mild, and A. Salo (2008). Robust portfolio modeling with incomplete cost information and project interdependencies. European Journal of Operational Research 190(3), 679-695.

Mancuso, A., M. Compare, A. Salo, E. Zio, and T. Laakso (2016). Risk-based optimization of pipe inspections in large underground networks with imprecise information. Reliability Engineering and System Safety 152, 228-238.

Mild, P., J. Liesiö, and A. Salo (2015). Selecting infrastructure maintenance projects with robust portfolio modeling. Decision Support Systems 77, 21-30.

Mustajoki, R. P. Hämäläinen, and A. Salo (2005). Decision support by interval smart/swing -incorporating imprecision in the smart and swing methods. Decision Sciences 36(2), 317-339.

Sacco, T., M. Compare, E. Zio, and G. Sansavini 
Table 1.: Comparison of failure likelihood and number of failures, 500 branches with largest $\bar{V}_{L}(b)$

\begin{tabular}{ccccccccc}
\hline Period & $1-50$ & $51-100$ & $101-150$ & $151-200$ & $201-250$ & $251-300$ & $301-400$ & $401-500$ \\
Percentage of failures & 0.073 & 0.055 & 0.01 & 0.045 & 0.041 & 0.027 & 0.032 & 0.027 \\
Cumulative percentage & 0.073 & 0.128 & 0.138 & 0.183 & 0.224 & 0.251 & 0.283 & 0.31 \\
\hline
\end{tabular}

(2019). Portfolio decision analysis for riskbased maintenance of gas networks. Journal of Loss Prevention in the Process Industries 60, 269-281.

Salo, A., J. Keisler, and A. Morton (2011). Portfolio decision analysis: improved methods for resource allocation, Volume 162. Springer Science \& Business Media.

Salo, A. A. and R. P. Hämäläinen (1992). Preference assessment by imprecise ratio statements. Operations Research 40(6), 1053-1061. 